\title{
Eccrine porocarcinoma: a case report and brief review of the literature
}

\author{
Rana Rafiei', Hojat Eftekhari², Arash Daryakar ${ }^{3}$, Nahid Nickhah², Behnam Rafiee $^{4}$ \\ ${ }^{1}$ Fellow ship of Dermatopathology, Skin Research Center, Guilan University of Medical Sciences, Razi Hospital, Rasht, Iran, \\ ${ }^{2}$ Skin Research Center, Guilan University of Medical Sciences, Razi Hospital, Rasht, Iran, ${ }^{3}$ Gastrointestinal and Liver Research \\ Center, Guilan University of Medical Sciences, Razi Hospital, Sardare Jangal St., Rasht, Iran, ${ }^{4}$ University of Texas, \\ MD Anderson Cancer Center, 1515 Holcombe blvd. B4.4512, Houston, TX, 77030, USA
}

Corresponding author: Rana Rafiei, MD., E-mail: rafieirana@yahoo.com

\begin{abstract}
Eccrine Porocarcinoma is a rare, slow growing malignant tumor of eccrine sweat gland. The most common site of this tumor is lower extremity. It is characterized histologically by anaplastic cells with eccrine ductal structures which may be adjacent to a benign eccrine poroma. We present here a case of Eccrine Porocarcinoma in lower extremity, with a brief review of the literature.
\end{abstract}

Key words: Eccrine carcinoma; Malignant eccrine poroma; Porocarcinoma; Eccrine poroma

\section{INTRODUCTION}

Eccrine Porocarcinoma (EPC) was first described by Pinkus and Mehregan in 1963 [1]. It is a rare malignant tumor but a fairly common type among eccrine carcinomas [2]. EPC may arise denovo but often develops from a benign eccrine poroma [3]. It usually presents on the extremities and head in old patients as asymptomatic, reddish, ulcerated nodule or plaque [4]. In $60 \%$ of cases EPC lesions occur on the lower extremities [1]. Trauma and occupational radiation exposure may have a role in tumor development $[5,6]$.

Special immunohistochemical (IHC) staining is essential for a definite diagnosis [1]. EPC may be purely intraepidermal or invasive. Lymphovascular invasion and distant metastasis could be seen in cases of the Invasive tumors. High mitotic rates, presence of lymphovascular invasion and tumor thickness more than $7 \mathrm{~mm}$ could be associated with poor prognosis [7].

\section{CASE REPORT}

A 64-year-old male was visited in dermatology outpatient clinic at Razi Hospital, Rasht, Iran. He had a dome-shaped reddish nodule without any symptoms or bleeding tendency on medial aspect of his right lower leg which first appeared 8 months ago. In addition there was an inflamed scaly erythematous nodule adjacent to it (Fig. 1).

In clinical examination there were two nodules: a $3 \mathrm{~cm} \times 3 \mathrm{~cm}$ reddish scaly firm nodule and a $1.5 \mathrm{~cm} \times$ $1.5 \mathrm{~cm}$ soft nodule without pigmentation or pain, about $5 \mathrm{~cm}$ above the right medial malleus.

Our patient had no history of trauma, radiation or contact with agrotoxic agents. He was doing well and the lesions developed denovo without any preexisting lesion in this region. The patient had a history of topical antibiotic therapy with no improvement.

In physical examination there was no organomegaly or lymphadenopathy. Clinical differential diagnoses were squamous cell carcinoma, melanoma, adnexal tumors and metastatic lesions. Skin biopsy was made from two sites: the larger firm nodule and the smaller inflamed nodule.

Pathological study of the larger lesion revealed a neoplastic skin tissue with overlying elongated epidermis composed

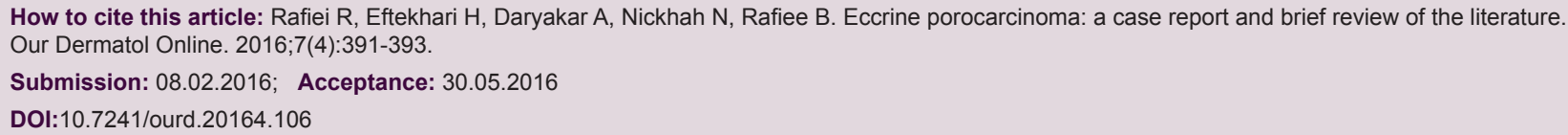


of interconnection strands of atypical epithelial poroid tumor cells with large vesicular nuclei, prominent nucleoli and acidophilic cytoplasm bearing cytoplasmic vacuolization, PAS positive hyaline globule like structures, cystic and duct like spaces without decapitation secretion, focal squamous differentiation with pushing border down to deep dermis. Numerous mitoses were evident. Surrounding dermis showed prominent plasmacytic infiltration (Figs. 2a and b).

In IHC study tumor cells were diffusely positive for pancytokeratin. Carcinoembryonic antigen (CEA) was positive more in ductal and cystic structures. Epithelial membrane antigen (EMA) was positive in vacuolated as well as ductal and cystic structures (Figs. 2 c-e). S100 and HMB45 were negative in tumor cells. All of these

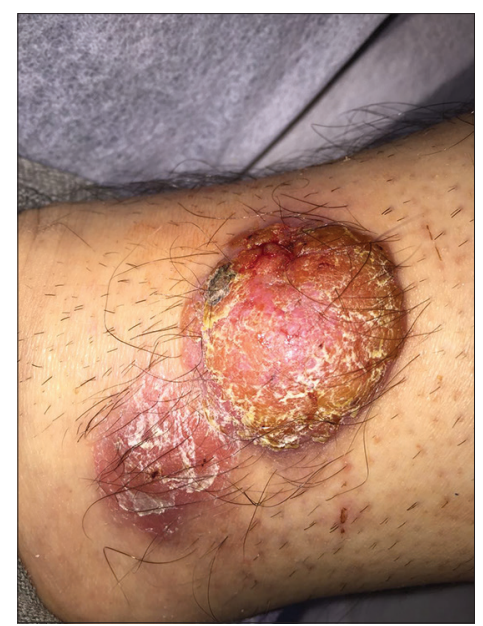

Figure 1: Two dome-shaped reddish nodules on medial aspect of the right lower leg. findings were more compatible with EPC. Pathologic study of the smaller lesion was more compatible with inflamed eccrine poroma.

Other laboratory tests and ultrasound examinations of the abdomen and pelvis were normal. Past medical history was negative. Tumoral lesion was excised with a safe margin of 15 millimeters and skin graft was done by a plastic surgeon. Pathologic study after total excision reported tumor free margins with pushing border down to deep dermis without subcutaneous involvement, so sentinel lymph node biopsy was not done. After 6 months follow up the patient was doing well without any recurrence.

Prior to the study, patient gave written consent to the examination and biopsy after having been informed about the procedure.

\section{DISCUSSION}

Our patient was interesting because he was simultaneously involved with benign eccrine poroma and EPC on the lower extremity. EPC is a sweat gland carcinoma and arise from the lower portion of intraepidermal eccrine ducts [8]. EPC incidence is estimated between $0.005 \%$ and $0.01 \%$ of cutaneous tumors. Benign eccrine poroma is more common on the palms and soles which have the highest concentration of eccrine glands, contrary to EPC which usually occurs on the lower extremity (44\%), trunk (24\%) or head $(18 \%)$. EPC diagnosis is dependent on either significant

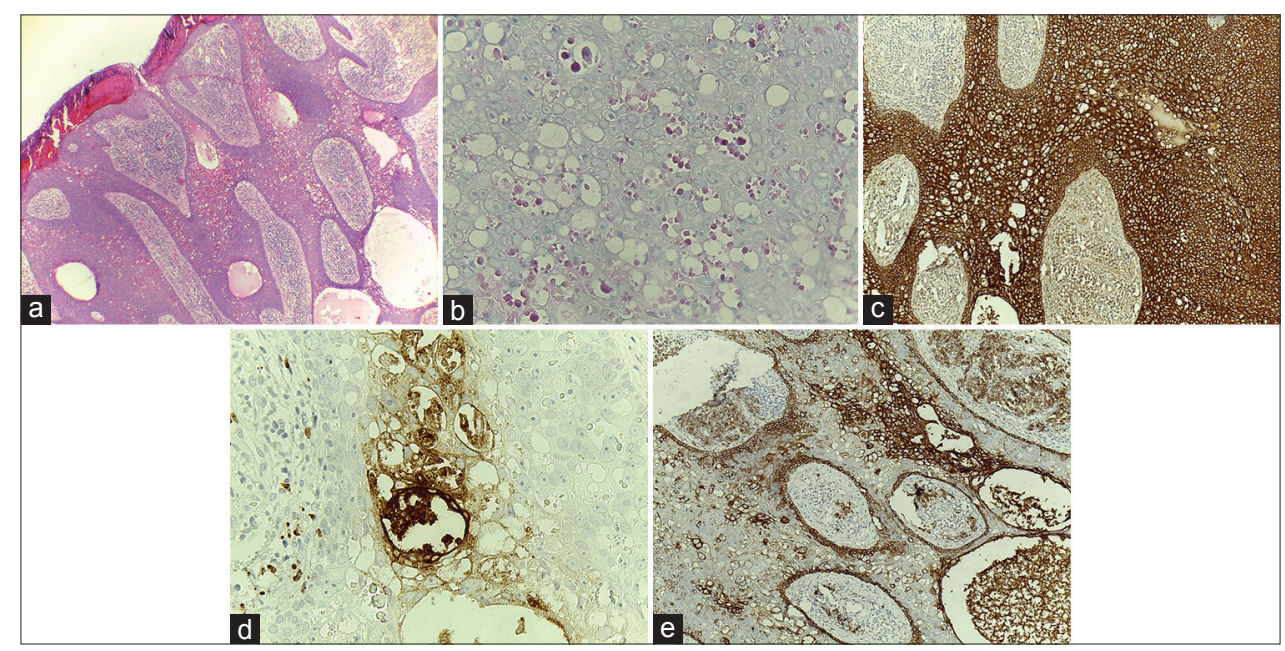

Figure 2: $(a, b)$ Elongated epidermis composed of interconnection strands of atypical epithelial poroid tumor cells with PAS positive hyaline globule like structures, cystic and duct like spaces with pushing border down to deep dermis. (A: hematoxylin-eosin, original magnification $\times 10-B$ : PAS staining, original magnification $x$ 40). (c) In IHC study tumor cells were diffusely positive for pan cytokeratin. (Original magnification $x$ 10). (d) In IHC study, CEA was positive more in ductal and cystic structures. (Original magnification x 40). (e) In IHC study, EMA was positive in vacuolated as well as ductal and cystic structures. (Original magnification $\mathrm{x} 10$ ). 
cytologic pleomorphism or an invasive growth pattern in a tumor with eccrine differentiation. Presence of eccrine type ducts is mandatory for diagnosis of EPC [7]. It often develops from or adjacent to a preexisting benign eccrine poroma with a longstanding history or even from a seborrheic keratosis $[1,3,7]$ so all benign poromas should be totally removed (1), but in one case report, all cases developed denovo [8]. It is estimated that $18 \%$ of poromas go on to change to EPC after a mean of 8.5 years [3]. It was interesting to us because our patient did not have any preexisting lesion and both lesions (EPC and benign inflamed poroma) developed simultaneouly eight months before the first visit. EPC might be entirely epidermal or could be able to extend in to dermis; which both of them have cystic lumina [1]. The prognosis of this tumor is still questionable but recent reports have predicted more indolent behaviors [8]. Several metastatic tumors have been reported especially in primarily misdiagnosed cases [9-11]. In another report, half of the patients had inaccurate primary diagnosis [12].The most important differential diagnoses of EPC are inflamed poroma, Bowen's disease, Paget's disease, amelanotic melanoma, metastatic adenocarcinoma. IHC studies with using antibodies to CEA and EMA should be performed for detecting ductal structures and PAS staining should be done for detecting PAS-positive cuticle to rule out metastatic adenocarcinoma [1]. In a clinicopathologic study of $69 \mathrm{EPC}$ cases mature well-formed eccrine ducts with eosinophilic luminal cuticle were observed in 68\% of cases and immature intracytoplasmic lumina was reported in $32 \%$ of cases. Lymphovascular invasion was present in $15 \%$ of cases [7]. Squamous differentiation (5\% of cases) and keratin pearl formation were reported in EPC, similarly to squamous cell carcinoma $[1,7]$. In case of our patient squamous differentiation, ductal formation and intracytoplasmic lumina were seen, and the tumor had pushing border in to the dermis but there were not any infiltrative growth pattern and lymphovascular invasion.

The gross size of a tumor has no significant association with the prognosis [12]. Tumor depth greater than $7 \mathrm{~mm}$, mitotic rate higher than 14 mitoses per high power field, infiltrative growth pattern and lymphovascular invasion are indicators of poorer prognosis $[1,7]$. EPC is usually treated by surgical excision with a safe margin of approximately $2 \mathrm{~cm}$ [8], which is $80 \%$ curative [2]. Due to lymphatic spread of EPC, sentinel lymph node biopsy might help us to detect subclinical lymph node involvement [8] but the role of lymphadenectomy is still questionable [2]. Chemotherapy and radiation have no place in treating metastatic disease [9].

\section{Consent}

The examination of the patient was conducted according to the Declaration of Helsinki principles.

\section{Abbreviations and Acronyms}

(EPC): Eccrine Porocarcinoma; (IHC): Immuno histochemical; (CEA): Carcinoembryonic antigen; (EMA): epithelial membrane antigen; (CK): Cytokeratin; (PAS): Periodic acid-Schiff; (HMB45): Human melanoma black 45

\section{REFERENCES}

1. Brown Jr CW, Dy LC. Eccrine porocarcinoma. Dermatologic therapy, 2008;21:433-8.

2. Lozano Orella JA, Valcayo Penalba A, San Juan CC, Vives Nadal R, Castro Morrondo J, Tunon Alvarez T. Eccrine Porocarcinoma: Report of Nine Cases. Dermatol Surg. 1997;23:925-8.

3. Sawaya JL, Khachemoune A. Poroma: a review of eccrine, apocrine, and malignant forms. Int J Dermatol. 2014;53:1053-61.

4. Bottles K, Sagebiel RW, McNutt NS, Jensen B, Deveney K. Malignant Eccrine Poroma; Case Report and Review of the Literature. Cancer. 1984;53:1579-85.

5. Requena L, Sanchez M, Aguilar A, Ambrojo P, Yus ES. Malignant metastatic eccrine poroma.Dermatologica. 1990:180:177-80.

6. Ruffliex C, Ramelet A. Porocarcinoma eccrine. Dermatologica 1985:170:202-6.

7. Robson A, Greene J, Ansari N, Kim B, Seed PT, McKee PH, et al. Eccrine porocarcinoma (malignant eccrine poroma): a clinicopathologic study of 69 cases. Am J Surg Pathol. 2001;25:710-20.

8. Shiohara J, Koga H, Uhara H, Takata M, Saida T. Eccrine porocarcinoma: clinical and pathological studies of 12 casesJ Dermatol. 2007;34:516-22.

9. Marone U, Caracò C, Anniciello AM, Di Monta G, Chiofalo MG, Di Cecilia ML, et al. Metastatic eccrine porocarcinoma: report of a case and review of the literature.World J Surg Oncol. 2011;9:32.

10. Goel R, Contos MJ, Wallace ML. Widespread metastatic eccrine porocarcinoma. J Am Acad Dermatol. 2003;49 (5 Suppl):S252-4.

11. Barzi AS, RuggeriS, Recchia F, Bertoldi I. Malignant metastatic eccrine poroma. Dermatol Surg. 1997;23:267-72.

12. Luz Mde A, Ogata DC, Montenegro MF, Biasi LJ, Ribeiro LC. Eccrine porocarcinoma (malignant eccrine poroma): a series of eight challenging cases. Clinics (Sao Paulo). 2010;65:739-42.

Copyright by Rana Rafiei, et al. This is an open access article distributed under the terms of the Creative Commons Attribution License, which permits unrestricted use, distribution, and reproduction in any medium, provided the original author and source are credited.

Source of Support: Nil, Conflict of Interest: None declared. 